Bull. Mater. Sci. Vol., 2, Number 5, December 1980, pp. 353-356. (C) Printed in India.

\title{
Theory of susceptibility of a-Ge and a-Si
}

\author{
A MOOKERJEE and S C AGARWAL \\ Department of Physics, Indian Institute of Technology, Kanpur 208 016, India \\ MS received 4 February 1980
}

\begin{abstract}
We propose a model of the thin films of a-Ge and a-Si with microvoids as frustrated spin glasses. Within this model the various qualitative aspects of the experimental results can be reasonably explained.
\end{abstract}

Keywords. Magnetic properties; amorphous semiconductors.

Some time ago Fritzsche and Hudgens (1975) and Hudgens (1976) measured the static magnetic susceptibility of tetrahedrally co-ordinated amorphous semiconductors a-Ge and a-Si (see figure 1). These materials are known to contain microvoids and dangling bonds which give rise to free spins. The density of these spins have been measured by ESR and is found to depend on the method of preparation and the state of anneal for each material, Agarwal (1973).

The spin component of the susceptibility $\chi_{S}$, which is found by subtracting the weakly temperature-dependent diamagnetic lattice contribution $\chi_{L}$ from the total measured susceptibility, obeys a Curie-Weiss law of the type $\chi_{s}^{-1} \alpha\left(T+\theta_{N}\right)$ for high temperatures. This is very similar to the behaviour of antiferromagnetic

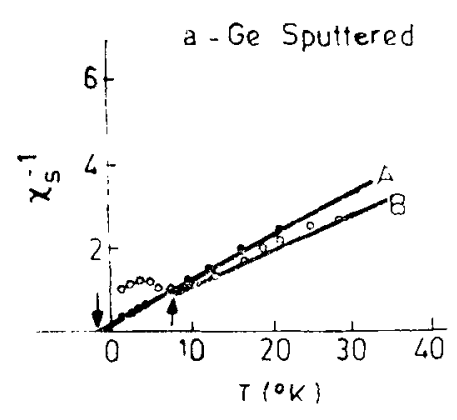

(a)

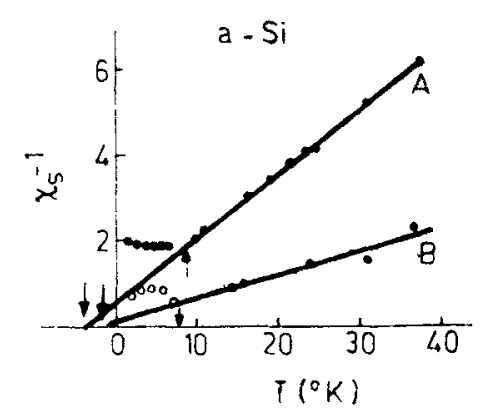

(b)

Figure 1a. Spin susceptibility of sputtered a-Ge. Curve $\mathrm{A}$ : in pure $\mathrm{Ar}+10^{-4}$ torr $\mathrm{H}_{2}$. Curve $\mathrm{B}$ : in pure Ar. 1b. Spin susceptibility of a-Si. CurveA : Sputtered in pure Ar. Curve B: Glow discharged. Data from Fritzscheand Hudgens (1975). Arrows $\uparrow$ and $\downarrow$ indicate $T_{N}$ and $\theta_{N}$ respectively. 
materials. When the temperature is lowered below an "ordering" temperature $T_{N}$, the susceptibility begins to show a striking departure from the Curie-Weiss behaviour indicating a magnetic ordering of some sort. Further, $T_{N}$ and $\theta_{N}$ both depend upon the state of the anneal and the method of preparation of each sample. Both decrease on annealing the as-deposited thin film below its crystallization temperature. For all samples and states of anneal $T_{N}>\theta_{N}>0$. $\chi_{s}$ definitely has a maximum at $T_{N}$; whether it has a cusp or not is not very clear from the experimental data, because of the difficulty in performing low field susceptibility experiments. But the indications are that it has.

We should like to suggest that the experimental data are consistent with the hypothesis that these systems order like spin glasses, with the spin glass like behaviour arising out of a "frustration" or "blocking" effect of the distorted lattice of the amorphous film.

There is a major difference between these systems and the usual metallic antiferromagnetic spin glass alloys (like $\mathrm{CuMn}$, for example). The number of conduction electrons in $\mathrm{a}-\mathrm{Ge}$ or a-Si are almost negligible in comparison with the metallic alloys. Hence, the RKKY interaction between the spins, mediated by the conduction electrons, cannot be invoked in these systems to explain the spin glass ordering. We must look for a different mechanism for the interspin interaction We propose that a superexchange interaction between the various cluster of spins situated on the voids may be responsible for the magnetic ordering. This superexchange arises because of the overlap of the localized electronic wave functions situated on the void sites. If the localization length of the electronic wave functions situated on the voids is not much smaller than the distance between the voids, this overlap may be significant and the resulting interaction not negligible. As is the case of all superexchange interactions arising out of wave function overlaps, it is easy to convince ourselves that the interaction must be antiferromagnetic.

Hudgens has considered several plausible explanations. However, none appears to be completely satisfactory. The non-interacting cluster viewpoint predicts $T_{N}<$ $\theta_{N}$, for all cases, which is contrary to the experimental results. Application of Southern's spin glass model is inadmissible, because the traditional spin glass theories require a fluctuating sign RKKY type of interaction, which cannot be invoked in our amorphous system. The correlation effects in the Hubbard band model cannot explain why $T_{N}, \theta_{N}$ change on annealing, whereas the ESR signal at low temperatures remains unchanged.

We propose an alternative plausible model and argue that it can explain all the features of the experimental results. Our model may be summarized as follows. It is known that structurally the thin films of a-Ge and a-Si are distorted tetrahedral networks. The models constructed by Polk (1971) serve as a gaod picture of the actual structure of the thin films. Unlike the perfect crystal, these distorted networks contain odd-membered rings: predominantly five and seven membered rings. The voids sit on the random network, forming a distorted "supernetwork" of voids against the network of $\mathrm{Ge}$ or $\mathrm{Si}$ atoms. Each void contains dangling bonds giving rise to spins which interact with each other, provided their distance apart are not much larger than the localization length of the wave functions localized on the voids. In a pure diamond lattice, this would give rise to an antiferromagnetic ordering. However, on our distorted lattice, 
because of the odd-membered rings there is a degree of "frustration", On none of the odd-membered rings can the spins order antiferromagnetically without ambiguity. Figure 2 illustrates this on a five-membered ring. These are precisely the "mismatches" mentioned by Mott (1968) when he referred to amorphous antiferromagnets. But in our case, far from being an embarrassing ignorable effect, these mismatches (i.e. frustrations or blockings) are crucial to the magnetic ordering. It has recently been argued both from first principles and from computer simulation data that such frustrated rings or plaquettes can lead to a spin glass phase with a characteristic cusp in the static spin susceptibility (Toulouse 1977; Kirkpatrick 1977). If the concentration of the frustrated rings is larger than a critical one, then the corresponding ordering temperature $T_{t}$ is higher than the antiferromagnetic ordering temperature, so that the system becomes a spin glass on cooling, before it has a chance of becoming an antiferromagnet. The Neel temperature is definitely positive and is proportional to the temperature equivalent of the coupling energy $J / k_{B}$. The susceptibility has a cusp at $T_{n}=$ $T_{N}>0_{N}$. The further decrease of $\chi_{s}{ }^{-1}$ below $T_{N}$ arises, as Hudgens suggested, due to a proportion of non-interacting spins. These, in our model, sit on those voids which are so far from their neighbours that the wave function overlap between them and their neighbours is very small and does not give rise to any appreciable superexchange interaction.

Within our model, the line width of the ESR signal is caused by the interaction of the dangling bond spins within a void, as suggested by Agarwal (1973), but the $T_{N}, \theta_{N}$ depend upon intervoid interactions. It is quite likely that annealing changes the intervoid interaction by changing the spatial distribution of the voids. Some voids may collapse and change the concentration of frustrated rings. This crucially alters the $T_{N}$ and $\theta_{N}$. However, the interspin distances within a single void may still remain the same, since it depends upon the way the voids change in shape on annealing.

The! ack of reliable data of susceptibility measurements and how $T_{N}, \theta_{N}$ change on annealing, the size and distribution of the voids in the amorphous films and the way these distort on annealing, prevent us from making any quantitative comparison between theory and experiment. Low angle $x$-ray scattering data can give crucial information on the shape, size and distribution of the voids. This, together with precise measurements of $T_{N}, O_{N}$ and the ESR results should permit us to test the validity of our model. Until these data are available, we propose this model as a plausible one, within which we can reasonably expect the quali-

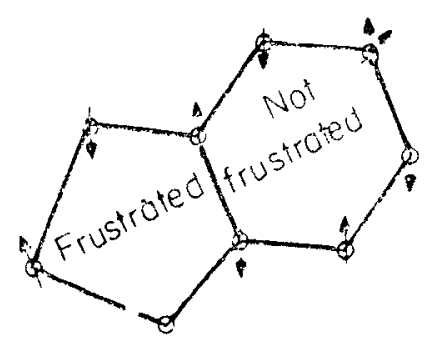

Figure 2. Spin frustration on a five-membered ring. 
tative behaviour as seen by experiment. These systems provide us with striking examples of real physical systems in which spin glass behaviour arises entirely due to the frustration effect.

\section{Acknowledgement}

The work was done under the project "Elestronic proparties of disordered solids" and was supported by the Department of Science and Technology, Government of India.

\section{References}

Agarwal S C 1973 Phys. Rev. B7 2481

Fritzsche H and Hudgens S J 1975 Liquid and amorphous semiconductors, Proc. Int. Conf., Leningrad p. 19

Hudgens S J 1976 Phys, Rev. B14 1547

Kirkpatrick S 1977 Phys. Rev. B16 4630

Mott N F 1968 Philos. Mag. 171259

Polk D E $1971 J$. Non-Cryst. Solids 5365

Toulouse G 1977 Commun. Phys. 2115 